\title{
The structural properties of transition metal hydrogen complexes in silicon
}

\author{
A. Resende ${ }^{\mathrm{a}, 1}$, R. Jones ${ }^{\mathrm{a}}$, S. Öberg ${ }^{\mathrm{b}, 2}$ and P. R. Briddon ${ }^{\mathrm{c}}$ \\ ${ }^{a}$ Department of Physics, The University of Exeter, Exeter, EX4 4QL, UK \\ b Department of Mathematics, University of Luleå, Luleå, S95 187, Sweden \\ ${ }^{\mathrm{c}}$ Department of Physics, The University of Newcastle upon Tyne, \\ Newcastle upon Tyne, NE1 7RU, UK
}

\begin{abstract}
A first-principles spin-polarised local density functional cluster method is used to explore the structural and vibrational properties of several substitutional transition-metal impurities complexed with hydrogen.
\end{abstract}

Key words: Ab initio theory, transition-metal impurities, H, Si.

\section{Introduction}

It is now a well-known fact that hydrogen can interact with transition-metal (TM) impurities, forming either electrically active or inactive complexes. An example of the former is the paramagnetic and orthorhombic $\left(\mathrm{PtH}_{2}\right)^{-}$defect, observed experimentally by electron-paramagnetic resonance and IR-absorption [4]. Despite the success with the $\mathrm{Pt}^{-}$centre, experimental information on the structure and chemical composition of other $\mathrm{TM}-\mathrm{H}_{n}$ complexes is still rather sparse and inconclusive.

\section{Methodology \& results}

Before presenting the results of the present study on the structural properties of TM$\mathrm{H}_{n}$ complexes in $\mathrm{Si}$, we describe the structure of the single substitutional TM impu-

${ }^{1}$ EPSRC and the ENDEASD network are thanked for computer time and financial support respectively.

2 Financial support by NFR and TFR in Sweden. PDC at KTH in Sweden is gratefully acknowledged for computer time on the SP2. 
rity. Non-relativistic spin-polarised local density functional theory is used, within a pseudo-potential real-space method (AIMPRO) to study the structural properties of substitutional, neutral gold $\left(\mathrm{Au}_{s}^{0}\right)$ and single negatively charged platinum $\left(\mathrm{Pt}_{s}^{-}\right)$, as well as the $\mathrm{TM}-\mathrm{H}_{n}$ complexes with $n=1$ and 2 for both impurities. Details on the method can be found elsewhere [3].

\subsection{Substitutional $\mathrm{Au}^{0}$ and $\mathrm{Pt}^{-}$}

For substitutional $\mathrm{Pt}^{-}$and $\mathrm{Au}^{0}$, there are three electrons in the one-electron $t_{2}$ manifold, which makes this state Jahn-Teller sensitive. Experiment and theoretical studies have shown that the JT-stabilised structures have $\mathrm{C}_{2 v}$ symmetry, spin $S=1 / 2$ for a ground-state configuration $b_{2}^{\uparrow \downarrow} b_{1}^{\uparrow} a_{1}^{0}$, in agreement with the vacancy model [1]. According to this model, the electronic properties of substitutional TM impurities near the end of the $3 d, 4 d$ and $5 d$ series can be described by considering a neutral TM atom with a saturated $d^{10}$ shell occupying a negatively charged lattice vacancy. As a consequence, the structural and electronic properties of these impurities are dictated by the vacancy-like character of the $s p^{3} \mathrm{Si}$ hybrid orbitals and not by the many-electron character of the partially filled TM $n d$ shell.

The distortions have been investigated by breaking the symmetry and relaxing the Si inner atoms including the impurity, in a specific electronic configuration under the appropriate symmetry constraint [6]. The $\mathrm{C}_{2 v}$ structures with the impurity displaced off-site along the dihedral [100] direction have proven to be stable with small shifts of the neighbouring $\mathrm{Si}$ atoms. The magnitude of distortion is slightly larger for $\mathrm{Ni}_{s}^{-}$and $\mathrm{Pt}_{s}^{-}$than for $\mathrm{Au}_{s}^{0}$ or $\mathrm{Ag}_{s}^{0}$. The equilibrium structures were found to correspond to a displacement of $0.20 \AA$ for $\mathrm{Ni}^{-}$, and $0.15 \AA$ for $\mathrm{Pt}_{s}^{-}$. For $\mathrm{Au}_{s}^{0}$ and $\mathrm{Ag}_{s}^{0}$, these were 0.03 and $0.01 \AA$, respectively. Our calculations find a small barrier for thermal re-orientation, consistent with experimental results for $\mathrm{Pt}_{s}^{-}$and $\mathrm{Au}_{s}^{0}$. In the case of $\mathrm{Au}_{s}^{0}$ and $\mathrm{Pt}_{s}^{0}$, the thermal barrier was calculated to be 0.11 and $0.26 \mathrm{eV}$, respectively.

An alternative model has been introduced to explain apparent discrepancies between the observed $g$ values for $\mathrm{Pt}^{-}$and those predicted by the vacancy model for the same centre. To test the predictions of the two-neighbouring-bonding or dihedral model [2], the TM ion was displaced off-site by $0.8 \AA$ along the [100] direction, closer to two neighbouring $\mathrm{Si}$, and the opposite $\mathrm{Si}$ atoms to the vacancy were brought closer, to simulate the stronger tetragonal distortion. For $\mathrm{Pt}_{s}^{-}$, the TM ion moved back closer to its $\mathrm{T}_{d}$ site, to stabilise to within $0.01 \AA$ of the lattice site, in evident disagreement with the predictions of the dihedral model.

\section{$2.2 T M-H_{n}$ complexes}

Recent local vibrational-mode (LVM) spectroscopy studies [5] have revealed the existence of a gold-hydrogen defect, containing a single hydrogen atom in a $\mathrm{C}_{3 v}$ 
symmetry. Two local vibrational modes have been identified, at 1813.3 and 1827.1 $\mathrm{cm}^{-1}$, associated with two different charge states of the $\mathrm{AuH}_{1}$ complex. These bands shifted by 502.4 and $507.7 \mathrm{~cm}^{-1}$ when hydrogen was replaced by deuterium, demonstrating unequivocally the involvement of hydrogen in the defect. Samples containing an admixture of $\mathrm{H}_{2}$ and $\mathrm{D}_{2}$ exhibit no additional vibrational bands. Despite these facts, it was impossible to identify the atom to which the $\mathrm{H}$ atom is bonded, along the $<111>$ axis of the centre. However, recent studies indicate that the $\mathrm{AuH}_{1}$ might have a lower symmetry of $\mathrm{C}_{1 h}[5]$.

Table I. Hydrogen stretch modes for $\mathrm{AuH}_{1}$ complexes. Observed isotope shifts are given in parentheses. Values in $\mathrm{cm}^{-1}$.

\begin{tabular}{lccccccc}
$\left(\mathrm{AuH}_{1}\right)^{q}$ & $q=0$ & -1 & -2 & $\left(\mathrm{AuD}_{1}\right)^{q}$ & $q=0$ & -1 & -2 \\
\hline \multicolumn{7}{c}{ Expt. [5] $1813.3(1827.1) \& 1310.9(1319.4)$} \\
\hline $\mathrm{BC}$ & 2419.0 & 2500.8 & 2454.3 & $\mathrm{BC}$ & 1726.2 & 1783.5 & 1748.9 \\
$\mathrm{AB}_{\text {out }}^{\mathrm{C}_{1 h}}$ & 1947.4 & 1890.5 & 1893.2 & $\mathrm{AB}_{\text {out }}^{\mathrm{C}_{1 h}}$ & 1401.1 & 1359.6 & 1361.4 \\
$\mathrm{AB}_{\text {in }}^{\mathrm{C}_{1 h}}$ & 2014.5 & 1980.0 & 2118.0 & $\mathrm{AB}_{\text {in }}^{\mathrm{C}_{1 h}}$ & 1427.3 & 1402.8 & 1500.2
\end{tabular}

To study the structural and vibrational properties of the $\mathrm{AuH}_{1}$ defect, three structures were used, in three different charge states. These were: $a$ ) $\mathrm{BC}$ (with the $\mathrm{H}$ atom sitting bond-centred between the TM impurity and a $\mathrm{Si}$ atom; $b$ ) $\mathrm{AB}_{\text {out }}$ (the $\mathrm{H}$ atom at the anti-bonding site to one of the Si neighbours) and $c$ ) $\mathrm{AB}_{\text {in }}(\mathrm{H}$ is inside the vacancy along the [111] direction. A lower symmetry $\mathrm{AB}_{\text {out }}$ configuration was also considered, in which the bond between two of the vacancy Si neighbours was slightly reconstructed. The $\mathrm{BC}$ and $\mathrm{AB}_{\text {in }}$ structures of the $\mathrm{AuH}_{1}$ complex were found to be less stable than the $\mathrm{AB}_{\text {out }}$ by 0.47 and $0.23 \mathrm{eV}$, respectively; the $\mathrm{C}_{3 v}$ and $\mathrm{C}_{1 h} \mathrm{AB}_{\text {out }}$ structures where found to be degenerate in energy. The $\mathrm{Si}-\mathrm{H}$ length was $1.52 \AA$ for both $\mathrm{C}_{3 v}$ and $\mathrm{C}_{1 h}$. For $\mathrm{AB}_{i n}$, the $\mathrm{Au}-\mathrm{H}$ length was $1.60 \AA$. The calculated stretch LVM frequencies are given in Table I. In all the structures, the off-site displacement of the TM impurity atom is rather small. For $\mathrm{AB}_{i n}$, the Au moves off-site by $0.06 \AA$ along $[\overline{1} \overline{1} \overline{1}]$ and $0.02 \AA$ along the [111] for $\mathrm{AB}_{\text {out }}^{\mathrm{C}_{1 h}}$. The energy barriers for re-orientation between symmetric configurations were, $0.41 \mathrm{eV}$ for $\mathrm{AB}_{\text {out }}^{\mathrm{C}_{1 h}}$, and $0.23 \mathrm{eV}$ for $\mathrm{AB}_{i n}$. These barriers are probably reduced by tunnelling.

The calculated quasi-harmonic local-vibrational modes $(\mathrm{LVMs})$ for $\left(\mathrm{PtH}_{1}\right)^{-}$were 1830.0 and $1728.1 \mathrm{~cm}^{-1}$, for $\mathrm{AB}_{\text {out }}^{\mathrm{C}_{1 h}}$ and $\mathrm{AB}_{\text {in }}$, respectively. Again, as in the case of $\mathrm{AuH}_{1}$, the closeness between LVM does not identify the structure responsible for the vibrational band at $1897.2 \mathrm{~cm}^{-1}$ seen in hydrogenated Pt-doped Si and attributed to a $\left(\mathrm{PtH}_{1}\right)$ defect [4].

We now investigate $\left(\mathrm{PtH}_{2}\right)^{-}$and $\left(\mathrm{AuH}_{2}\right)^{0}$. The structure of $\left(\mathrm{PtH}_{2}\right)^{-}$is consistent with experiment and previous modelling. The $\mathrm{AB}\left(\mathrm{C}_{2 v}\right)$ configuration was found to be more stable than the competing $\mathrm{BC}$ by $0.40 \mathrm{eV}$ for $\left(\mathrm{PtH}_{2}\right)^{-}$and $0.53 \mathrm{eV}$ for 
$\left(\mathrm{AuH}_{2}\right)^{0}$. The Si-H lengths were 1.54 and $1.52 \AA$, for the Pt-H and Au-H related defects, respectively. For $\left(\mathrm{PtH}_{2}\right)^{-}$, the splitting between the calculated $\mathrm{A}_{1}$ (1763.1) and $\mathrm{B}_{1}$ (1756.2) modes was $\sim 7 \mathrm{~cm}^{-1}$, in fair agreement with the observed value of $19.4 \mathrm{~cm}^{-1}$ [4]. As expected, due to a shorter $\mathrm{Si}-\mathrm{H}$ length, the stretch mode frequencies for the $\mathrm{AB} \mathrm{AuH}_{2}$ defect were 151.0 and $156.4 \mathrm{~cm}^{-1}$ higher than the $\mathrm{Pt}$ analogue.

\section{Conclusions}

In conclusion, the calculations show that $\mathrm{Pt}_{s}^{-}$is unstable against a slight displacement along the $<100>$ direction. $\mathrm{Au}_{s}^{0}$ shows a similar structure, with a weaker JT distortion. For the TM- $\mathrm{H}_{1}$ defects, the $\mathrm{AB}_{\text {out }}$ configurations were energetically favoured but the differences in energies with the $\mathrm{AB}_{\text {in }}$ configurations are not large. We tentatively assign the observed $\mathrm{AuH}_{1}$ defect to the $\mathrm{AB}_{\mathrm{C}_{1 h}}^{\text {out }}$ structure. As in previous theoretical studies [6], we have confirmed the $A B$ structure as the most stable TM- $\mathrm{H}_{2}$ defect structure, in which the two $\mathrm{H}$ atoms lie outside the vacancy.

\section{References}

[1] G. D. Watkins, Physica 117B \& 118B, 1149 (1983).

[2] C. A. J. Ammerlaan and A. B. van Oosten, Physica Scripta T25, 342 (1989).

[3] To appear in Identification of Defects in Semiconductors, edited by M. Stavola and Semiconductors and Semimetals, treatise editors, R. K. Willardson, A. C. Beer and E. R. Weber (Academic Press).

[4] S. J. Uftring, M. Stavola, P. M. Williams and G. D. Watkins, Phys. Rev. B51, 9612 (1995).

[5] M. J. Evans, M. G. Gornstein and M. Stavola, Mat. Res. Symp. Proc. 442, 275 (1997); M Stavola, private communication.

[6] R. Jones, S. Öberg, J. Goss, P. R. Briddon and A. Resende, Phys. Rev. Lett. 75, 2734 (1995). 